MATTEO NACCI

Pontificia Università Lateranense

\title{
IL MODELLO “ENTE ECCLESIASTICO”: LA PROSPETTIVA STORICA
}

Sommario: -1 . Chiarimenti preliminari. -2 . Lo svolgimento dell'attività assistenziale della Chiesa nel tempo. - 3. Brevi note storiche sull'ente ecclesiastico ospedaliero: dall'esperienza giuridica medievale a quella moderna. -4 . Nota conclusiva.

\section{Chiarimenti preliminari}

Vorrei innanzitutto ringraziare i colleghi del Dipartimento Jonico di Taranto per aver voluto includere anche la prospettiva storica all' interno di questo nostro incontro ${ }^{1}$. Scelta condivisibilissima quando si pensa che la lettura del dato storico non solo determina un quid pluris culturale all'interno di un convegno ma, ecco l'elemento per me costitutivo, aiuta a comprendere il reale bisogno della comunità del tempo presente - traducendolo in norme 'giuste' - e, soprattutto, quello delle generazioni future.

Leggendo il titolo del mio intervento - Il modello "ente ecclesiastico": la prospettiva storica - si potrebbe pensare che la presenza odierna dello storico del diritto debba consistere in una lettura dell'ente ecclesiastico nelle diverse epoche storiche evidenziandone le peculiarità di tempo e di luogo. Tale approccio metodologico

\footnotetext{
${ }^{1}$ Conferenza tenuta il 3 dicembre 2015 in occasione del Convegno nazionale Modelli organizzativi e obiettivi di efficienza nel governo dell'impresa sanitaria organizzato dal Dipartimento Jonico di Sistemi giuridici ed economici del Mediterraneo dell’Università degli Studi di Bari - Sede di Taranto.
} 
ed operativo si rivelerebbe sicuramente poco efficace poiché solo sfogliando le pagine dei grandi dizionari enciclopedici - purtroppo sempre più impolverati nelle nostre biblioteche a vantaggio di meno affascinanti portali informatici - si trovano mirabili ricostruzioni storiche alla voce 'enti ecclesiastici', frutto dello studio e della ricerca di giuristi di primo piano ${ }^{2}$. Per non parlare, poi, delle numerosissime riflessioni scientifiche in tema di enti ecclesiastici, da sempre oggetto di studio dei cultori del diritto ecclesiastico ${ }^{3}$.

E allora, sperando di non disattendere le aspettative di coloro che mi hanno invitato e leggendo sinotticamente il titolo del mio intervento con quello dell'intero convegno - Modelli organizzativi e obiettivi di efficienza nel governo dell'impresa sanitaria - credo che dallo storico del diritto ci si aspetti, in questa sede, una ricostruzione dell'evoluzione storico-giuridica del 'modello' ente ecclesiastico

\footnotetext{
${ }^{2}$ Si veda, fra molti, F. Scaduto, Enti ecclesiastici, in: Digesto Italiano, X, Torino 1926, pp. 478-484; G. ForChielli, Enti ecclesiastici, in: Nuovo Digesto Italiano, V, Torino 1938, pp. 429-430; F. Finocchiaro, Enti ecclesiastici, II) Enti ecclesiastici cattolici, in: Enciclopedia Giuridica, XII, Roma 1989, pp. 1-15.

${ }^{3} \mathrm{Si}$ veda, ex multis, G. Stocchiero, Enti e beni ecclesiastici in Italia dopo il Concordato, Società Anonima Tipografica, Vicenza 1930; R. BACCARI, Il potere autonomico degli enti ecclesiastici, La Floridiana, Napoli 1943; T. Mauro, Il problema della nazionalità degli enti ecclesiastici, Giuffrè, Milano 1959; G. LezIroli, Enti canonici ed enti ecclesiastici, Giuffrè, Milano 1974; A. M. Punzi Nicolò, Gli enti nell'ordinamento canonico, Cedam, Padova 1983; S. BERLINGò, Enti e beni religiosi in Italia, Il Mulino, Bologna 1992; G. DAmmacco, Sistema concordatario e patrimonio ecclesiastico, Cacucci, Bari 1995; P. Floris, L'ecclesiasticità degli enti: standards normativi e modelli giurisprudenziali, Giappichelli, Torino 1997; P. Cavana, Enti ecclesiastici e controlli confessionali, Giappichelli, Torino 2002; A. Bettetini, Gli enti e i beni ecclesiastici, Giuffrè, Milano 2005; I. Bolgiani (ed.), Enti di culto e finanziamento delle confessioni religiose. L'esperienza di un ventennio (1985-2005), Il Mulino, Bologna 2007; G. Rivetti, La disciplina tributaria degli enti ecclesiastici. Profimi di specialità tra attività no profit of for profit, 2 ed., Giuffrè, Milano 2008; P. Cavana, Gli enti ecclesiastici nel sistema pattizio, 2 ed., Giappichelli, Torino 2011; O. Fumagalli Carulli, Matrimonio ed enti tra libertà religiosa e intervento dello Stato, V\&P, Milano 2012; A. Bettetini, Gli enti e i beni ecclesiastici. Art. 831, 2 ed., Giuffrè, Milano 2013; P. Clementi - L. Simonelli (edd.), L'ente ecclesiastico a trent'anni dalla revisione del Concordato, Giuffrè, Milano 2015.
} 
ospedaliero. Tale tema presenta non poche difficoltà legate al fatto che molto spesso si considera l'ente ecclesiastico ospedaliero connesso soltanto alle molte questioni problematiche concernenti la legislazione e l'attività sanitaria odierna dimenticandoci, purtroppo, che tali enti hanno una storia che affonda le origini almeno nell'esperienza giuridica medievale. Basti pensare, ad esempio, a una realtà sanitaria legata al territorio nel quale stiamo svolgendo il nostro incontro: l'Ente Ecclesiastico Ospedale Generale Regionale "F. Miulli" con sede ad Acquaviva delle Fonti, in Provincia di Bari, la cui origine risale al pieno Medioevo, il 1158, anno in cui vide la luce come "Ospedale dei soldati" ma aperto anche alla popolazione civile ${ }^{4}$.

Non essendo questa, però, la sede opportuna per analizzare i singoli casi di ente ecclesiastico ospedaliero e l'evoluzione storica di ciascuno - potrebbe essere piuttosto una parte di un più ampio progetto di ricerca -, ho pensato di impostare il mio intervento facendo alcuni cenni all'attività assistenziale della Chiesa nell'ordinamento italiano, sulla base di una acutissima lettura compiuta da Giuseppe Dalla Torre ${ }^{5}$, per poi delineare alcuni aspetti del cosiddetto diritto ospedaliero - all'interno del quale rientra anche il modello ente ecclesiastico ospedaliero - in prospettiva squisitamente storicogiuridica, fino alla codificazione piano-benedettina, e ritenendo validissimo punto fermo lo studio di Emilio Nasalli Rocca ${ }^{6}$. Prospettiva storica perché, ratione competentiae, del modello ente ecclesiastico nella legislazione odierna si occuperanno altri relatori ed inoltre è stato recentemente pubblicato un volume, nell'ambito di

\footnotetext{
${ }^{4}$ Si veda A. Liddi, L’ospedale Miulli: un esempio di religiosità volto al bene sociale, in: Ospedale Miulli. Rassegna Trimestrale 20 (1999), pp. 29 ss.

${ }^{5}$ G. DAlla Torre, L'attività assistenziale della Chiesa nell'ordinamento italiano. Aspetti dogmatici e spunti ricostruttivi, Giuffrè, Milano 1979. In tema di assistenza si veda, inoltre, M. C. Folliero, L'assistenza e la beneficenza tra legislazione e diritto costituzionale vivente, Edisud, Salerno 1990.

${ }^{6}$ E. NAsalli Rocca, Il diritto ospedaliero nei suoi lineamenti storici, Fondazione Sergio Mochy Onory per la storia del diritto italiano, Milano 1956.
} 
una ricerca in tema di regime giuridico degli enti ecclesiastici ${ }^{7}$, che affronta il tema dell'ente ecclesiastico ospedaliero ${ }^{8}$.

\section{Lo svolgimento dell'attività assistenziale della Chiesa nel tempo}

In apertura del testo dapprima menzionato, Giuseppe Dalla Torre esprime un concetto che di per sé esaurirebbe una buona parte di contenuto del mio intervento e, al tempo stesso, profilerebbe le linee direttrici del tempo presente. L'insigne canonista, infatti, osserva che «la Chiesa ha avuto il merito di sviluppare nel corso dei secoli teorie e prassi assistenziali, che costituiscono il retroterra comune e necessario dell'odierno bagaglio culturale, professionale e tecnico in materia di servizi sociali» ${ }^{9}$. Il punto fondamentale è questo. La Chiesa, dal punto di vista dello storico del diritto, ha avuto un ruolo determinante nello sviluppo della società umana tanto più protagonistico quanto più era assente l'apparato statuale di riferimento. Essa, cioè, aveva un indiscusso ruolo di primo piano all' interno della società medievale in cui il potere politico si presentava incompiuto e il legislatore era quasi del tutto assente. 'Incompiutezza del potere politico' e 'appartatezza del legislatore' sono due espressioni che rendono perfettamente l'idea del vuoto politico in cui vive il mondo medievale e del ruolo di supplenza svolto egregiamente dalla Chiesa, anche sotto il profilo dell'assistenza: di fronte ad un principe occupato quasi esclusivamente a difendere

\footnotetext{
${ }^{7}$ M. C. Folliero, Enti religiosi e non profit tra Welfare State e Welfare Community. La transizione, Giappichelli, Torino 2010. Si veda, altresì, M. C. Folliero, L'assistenza e la beneficenza tra legislazione e diritto costituzionale vivente, Edisud, Salerno 1990; G. CAsusCElli, Enti ecclesiastici e doveri di solidarietà, in: Stato, Chiese e pluralismo confessionale. Rivista telematica (www.statoechiese.it), 7 (2014), pp. 1-54.

${ }^{8}$ C. Elefante, Enti ecclesiastici ospedalieri, sanità pubblica e spending review, Giappichelli, Torino 2014.

${ }^{9}$ G. Dalla Torre, L'attività assistenziale della Chiesa nell'ordinamento italiano. Aspetti dogmatici e spunti ricostruttivi, p. 3.
} 
i confini dei suoi domìni e a riscuotere tasse, essa mette a disposizione la sua esperienza umana e giuridica per la società medievale ${ }^{10}$.

Ed è proprio questo maggior ruolo della Chiesa - soprattutto in epoca medievale ma dal punto di vista istituzionale già quando s'iniziò a concedere alla stessa la possibilità di vivere come societas licita - che è dato ammirare nella sua vocazione, da sempre presente, di prestare il proprio servizio e la propria assistenza, anche di tipo ospedaliero, alle persone bisognose e sofferenti ${ }^{11}$. Da questo momento, grazie soprattutto alla sensibilità della Chiesa, si profila una serie di diritti legati ai poveri, i cosiddetti iura pauperum, la cui tutela vedrà un'alternanza variabile a seconda che si consideri l'esperienza giuridica medievale, nella quale essi troveranno la loro più completa attuazione, o la modernità giuridica. Nei tempi moderni, infatti, la matrice individualistica - tanto socio-antropologica quanto giuridico-normativa - unita alla secolarizzazione prenderà sempre più spazio determinando una progressiva messa all'angolo della Chiesa come fornitrice di assistenza (anche ospedaliera) a favore di un ruolo sempre maggiore dello Stato nel garantire tale servizio. Ruolo sancito, in questa epoca pos-moderna, tanto a livello nazionale nelle carte costituzionali - si pensi all'articolo 38 della Costituzione della Repubblica italiana $^{12}$ - quanto a livello sovranazionale ${ }^{13}$.

In base a quanto sinteticamente osservato, si può trarre una prima conclusione che è anche angolo di osservazione proprio ed autonomo dello svolgimento storico della cosiddetta attività assistenziale legata ai due soggetti fornitori: la Chiesa e lo Stato. Ed è proprio all'interno

${ }^{10}$ P. Grossi, L'Europa del diritto, 7 ed., Laterza, Bari 2011, pp. 11-16; 27-29.

${ }^{11} \mathrm{G}$. DAlla Torre, L'attività assistenziale della Chiesa nell'ordinamento italiano. Aspetti dogmatici e spunti ricostruttivi, pp. 3-6.

${ }^{12} \mathrm{Si}$ veda, sul punto, O. SEPE, Il diritto all'assistenza nella Costituzione, Giuffrè, Milano 1959; G. Licheri, Costituzione e assistenza sociale, M. Ragno, Roma 1978; V. Colalillo, La tutela della salute nella Costituzione italiana, Jovene, Napoli 1979; L. Dimasi, Il c.d. dovere della salute, EDAS, Messina 1986; C. BotTARI, Principi costituzionali e assistenza sanitaria, Giuffrè, Milano 1991.

${ }^{13}$ Si rimanda alle osservazioni di G. DAlla TORRE, L'attività assistenziale della Chiesa nell'ordinamento italiano. Aspetti dogmatici espunti ricostruttivi, p. 7, nota 6. 
della considerazione del quantum di assistenza fornito dalla Chiesa e dalle comunità politiche territoriali che si può evidenziare un elemento di frizione fra l'una e le altre, come giustamente sostiene Dalla Torre quando afferma che è «indubbio che l'assistenza rientri fra le materie tipiche dello scontro fra la Chiesa e lo Stato moderno (...), uno scontro che comporta fra l'altro, per più ragioni l'estromissione della Chiesa dal campo assistenziale e che se sul piano della prassi politica e normativa vede lo Stato imporre coattivamente la propria supremazia, nel quadro di un sistema giurisdizionalista, o di uno statualismo separatista, ovvero lo vede scendere a trattative con la Chiesa per addivenire ad una necessaria actio finium regundorum, sul piano della speculazione politica e giuridica conduce, da una parte e dall'altra, alla ricerca ed alla affermazione (...) di motivazioni atte a sostenere le reciproche posizioni e rivendicazioni ${ }^{14}$.

Ė anche vero, però, come sottolinea Dalla Torre, che nello svolgimento dell'attività assistenziale, a volte teso, tra la Chiesa e lo Stato, la prima abbia sempre mostrato una indubbia 'adattabilità'. E la caratteristica dell'adattabilità della Chiesa in relazione all'attività assistenziale significa che essa, nel corso della storia, ha saputo sempre organizzarsi e adattarsi in base alle circostanze di tempo e di luogo riuscendo a garantire, seppure in mezzo a molte avversità - tanto politiche, ad esempio i governi nazionalisti, quanto climatiche, basti ricordare le virulenti pestilenze medievali - una efficace, efficiente e misericordiosa attività assistenziale ${ }^{15}$. La Chiesa è sempre riuscita a tenere vivo e ad applicare lo spirito della caritas cristiana e a fare in modo che esso, anche nei periodi più bui, fosse un lume di speranza e di forza per la compagine sociale di ogni tempo e luogo: dall'amministrazione in epoca medievale della quarta pauperum

\footnotetext{
${ }^{14}$ G. Dalla Torre, L'attività assistenziale della Chiesa nell'ordinamento italiano. Aspetti dogmatici e spunti ricostruttivi, p. 9. Per un'analisi dei rapporti fra potere religioso e potere civile si vedano, in particolare, le pp. 18-32 del volume di Dalla Torre menzionato e la qualificata bibliografia ivi citata.

${ }^{15}$ G. DAlla Torre, L'attività assistenziale della Chiesa nell'ordinamento italiano. Aspetti dogmatici e spunti ricostruttivi, p. 36.
} 
dei redditi ecclesiastici alle numerose attività caritative svolte dalla Chiesa in questo tempo pos-moderno.

\section{Brevi note storiche sull'ente ecclesiastico ospedaliero: dall'esperienza giuridica medievale a quella moderna}

Vediamo ora in che modo l'attività assistenziale della Chiesa, fin qui delineata in modo sintetico, si sia tradotta materialmente nella strutturazione di enti ecclesiastici ospedalieri lungo il divenire dell'esperienza giuridica medievale e moderna. Nel corso dell'esperienza giuridica medievale - prescindendo in questa sede da un'analisi degli avvenimenti del mondo romano (in modo particolare l'Editto di Licinio e Costantino del 313 d.C. e l'Editto di Tessalonica del 380 d.C. $)^{16}$ determinanti la genesi di una organizzata attività assistenziale ospedaliera ecclesiastica come diretta conseguenza della possibilità per la Chiesa di possedere e amministrare beni materiali alla luce del sole - lo sviluppo degli enti ecclesiastici ospedalieri si deve senzombra di dubbio a due fattori: la caratteristica intrinseca

${ }^{16}$ Fra le numerosissime pagine scritte sull'Editto di Licinio e Costantino del 313 d.C. si veda, G. Lombardi, Persecuzioni, laicità, libertà religiosa. Dall'Editto di Milano alla "Dignitatis humanae", Studium, Roma 1991; E. DAL Covolo, Chiesa. Società. Politica. Aree di laicità nel cristianesimo delle origini, LAS, Roma 1994, pp. 17-30; E. DAL Covolo, Il «capovolgimento» dei rapporti tra la Chiesa e l'Impero, in: E. dal Covolo-R. Uglione (edd.), Chiesa e Impero da Augusto a Giustiniano, LAS, Roma 2001, pp. 199-208; G. BonAmEnte, La «svolta costantiniana», in: E. dal Covolo-R. Uglione (edd.), Chiesa e Impero da Augusto a Giustiniano, LAS, Roma 2001, pp. 145-170; G. RinAldi, Ridurre a minoranza. Riflessione su alcuni percorsi dei pagani nell'impero dei cristiani, in: A. Zanbarbieri-G. Otranto (edd.), Cristianesimo e Democrazia, Edipuglia, Bari 2011, pp. 135-147; E. DAL Covolo, Eziologia storico-religiosa della cosiddetta "svolta costantiniana", Salesianum 75 (2013), pp. 537-546; M. NAcci, Il concetto di "libertà religiosa” all'indomani dell'editto di Licinio e Costantino: brevi considerazioni storico-giuridiche, Revista General de Derecho Romano 24 (2015), pp. 1-12. Sull'Editto Cunctos populos in relazione all'attività legislativa imperiale e al funzionamento della corte si veda il recente contributo di L. De Giovanni, Imperatori, corti, attività legislativa nella tarda antichità, in: Centro Italiano di Studi sull'Alto Medioevo (ed.), Le corti nell'Alto Medioevo, Atti delle Settimane di Studio (Spoleto, 24-29 aprile 2014), Fondazione Centro Italiano di Studi sull'Alto Medioevo, Spoleto 2015, pp. 357-384. 
dell'esperienza giuridica medievale da una parte, il concetto di universitas dall'altra.

Circa il primo, ho già avvertito come nel Medioevo il maggior ruolo della Chiesa nel campo dell'assistenza ospedaliera derivi dalle trame più intime dello stesso tempo medievale; un tempo in cui il detentore del potere politico appare piuttosto indifferente alla produzione del diritto ed è interessato, viceversa, esclusivamente al mantenimento dell'esercito e alla riscossione delle tasse. In tale panorama la Chiesa supplisce all'assenza del potere politico sia attraverso la sua organizzazione capillare che raggiunge le più remote campagne, sia grazie alla cospicua proliferazione di congregazioni religiose con finalità assistenziali-ospedaliere.

Il secondo fattore di sviluppo degli enti ecclesiastici ospedalieri il concetto di universitas - rappresenta anch'esso una delle cifre più intime del mondo medievale. Sono l'insieme delle universitates che formano quei centri giuridici autonomi, fra i quali anche gli ospedali ${ }^{17}$, che caratterizzano il pluralismo e il comunitarismo medievale ${ }^{18} \mathrm{e}$ dove è possibile in ogni caso individuare una certa tensione tra la Chiesa e le comunità politiche in relazione allo svolgimento dell'assistenza ospedaliera. Fra gli ospedali medievali costituiti sotto forma di universitas, degni di menzione sono gli Ospedali per i lebbrosi dedicati a San Lazzaro, realtà in cui era forte la presenza del vescovo che doveva proporre e giudicare le condizioni di ammissibilità per l'ingresso nell'ospedale ma in cui i Comuni svolgevano un'importante attività d'ordine pubblico (la determinazione del luogo nel quale costruire il lebbrosario e della distanza di sicurezza dal centro urbano in modo da evitare contagi con il resto della popolazione $)^{19}$.

\footnotetext{
${ }^{17}$ Sul concetto di universitas ospedaliera in epoca medievale si rinvia a E. NASALLI Rocca, Il diritto ospedaliero nei suoi lineamenti storici, pp. 52-62.

${ }^{18}$ Sul ruolo delle comunità intermedie nell'esperienza giuridica medievale si veda P. Grossi, Le comunità intermedie tra moderno e pos-moderno, Marietti, Genova 2015.

${ }^{19}$ E. Nasalli Rocca, Il diritto ospedaliero nei suoi lineamenti storici, pp. 62-66 ed ivi ampia bibliografia citata.
} 
Particolarmente interessante è il modello gestionale degli Ospedali di San Lazzaro per i lebbrosi. Un modello gestionaleorganizzativo che vede i dirigenti dell'ospedale e i malati, i fratres e gli infirmi, collaborare insieme, anche se in una stretta gerarchia, nell'amministrazione dell'ospedale. Tanto i dirigenti quanto i malati costituivano un tutt'uno per il buon funzionamento dell'ospedale. Quindi un modello particolare di coordinamento fra i fornitori e i destinatari dell'attività assistenziale; un modello organizzativo che non troviamo in altre tipologie di ente ospedaliero di epoca medievale ${ }^{20}$.

Oltre agli ospedali costituiti sulla forma giuridica dell'universitas, il Medioevo conosce altri modelli ecclesiastici ospedalieri. Fra questi, degni di nota sono gli hospitia ecclesiastica. Tali enti ecclesiastici ospedalieri sorgevano soprattutto nei pressi dell'episcopio e rispondevano al dovere di investire la quarta pauperum dei redditi ecclesiastici ${ }^{21}$. Erano principalmente fondati dal vescovo ma, anche nel caso in cui non lo fossero, l'autorità ecclesiastica svolgeva comunque un forte potere di controllo che consisteva in precisi diritti e doveri: il diritto di ricevere il rendiconto delle spese affrontate dall'ente ospedaliero, il dovere di visita periodica dell'ente ospedaliero, il dovere di controllo dell'amministrazione economica. All'interno degli hospitia, a differenza degli ospedali giuridicamente costituiti sulla forma dell'universitas, il modello gestionale prevedeva la scissione tra organismi dirigenti e individui ai quali si indirizzava l'opera benefica in modo da garantire una maggiore autonomia dell'ospedale quale persona giuridica ${ }^{22}$.

La forte presenza della Chiesa nel Medioevo in relazione allo svolgimento di attività benefico-ospedaliera si profila anche attraverso una terza forma, oltre alle universitates e agli hospitia ecclesiastica. Faccio riferimento alla nascita e proliferazione di ordini religiosi sorti per occuparsi di attività assistenziale. Fra tutti, costituisce

\footnotetext{
${ }^{20} I v i$, pp. 5-6.

${ }^{21}$ Ivi, pp. 7-8.

${ }^{22}$ Ibidem.
} 
sicuramente un modello per gli ordini religiosi successivi l'Ordine Gerosolimitano Ospedaliero di San Giovanni. L'importanza di quest'ordine gerosolimitano deriva dal fatto che la sua attività si svolge sin dall'inizio lungo due linee direttrici. La prima riguardava l'assistenza ai pellegrini con riferimento alla cura delle strade e dei ponti; ed infatti, l'Ordine dei Frati toscani di Altopascio, una branca di questo ordine gerosolimitano, aveva il compito di garantire la buona percorrenza delle strade poiché tale servizio le rendeva più sicure e agibili e, conseguentemente, poteva essere determinante nel fornire una più celere e pronta assistenza a colui che ne abbisognava ${ }^{23}$. La seconda linea direttrice dell'assistenza fornita dall'ordine gerosolimitano, quella che a noi interessa più direttamente, è quella che si rivolgeva alla cura degli infermi, sulla base di un ordine nato precedentemente in Francia, l'Ordine di Santo Spirito, che aveva il compito di creare delle case di assistenza nelle grandi città, precursori dei grandi ospedali cittadini di epoca moderna ${ }^{24}$.

Nell'esperienza giuridica medievale, da quanto abbiamo potuto osservare, se da una parte legislazione e prassi canonica assorbono in modo preponderante l'ente ospedaliero determinando un ruolo di primo piano della Chiesa anche quando non è direttamente coinvolta nella sua fondazione, dall'altra si riesce quasi sempre a trovare una sorta di 'equilibrio gestionale' fra le due parti in causa (Chiesa e comunità politiche territoriali) nell'organizzazione dell'attività assistenziale ospedaliera ${ }^{25}$. Si pensi, ad esempio, alla legislazione di Clemente V, in occasione del Concilio di Vienne del 1311, dalla quale emerge chiaramente la superiore autorità vescovile nella gestione degli enti ospedalieri, anche di fondazione secolare, all'interno di quelle statuizioni che prevedevano il diritto di controllo del vescovo sulla gestione e buona amministrazione dell'ente ospedaliero o il divieto di conferire gli ospedali in beneficio ${ }^{26}$.

\footnotetext{
${ }^{23}$ E. Nasalli Rocca, Il diritto ospedaliero nei suoi lineamenti storici, p. 10; p. 78.

${ }^{24}$ Ivi, pp. 10-11; pp. 74-77.

${ }^{25}$ Ibidem.

${ }^{26}$ Ibidem.
} 
Questo 'equilibrio gestionale' è però destinato a cambiare profondamente nel passaggio dal Medioevo all'epoca moderna, un momento storico fortemente caratterizzato da un forte e spiccato individualismo. Un individualismo che investe tutti i campi: teologico, filosofico, antropologico, politico. Nella declinazione politica dell'individualismo, il principe moderno, detentore del potere e figura caratterizzante l'assolutismo monarchico, manifesta fin da subito una volontà totalizzante e centralizzatrice includendo nelle sue mani, per l'appunto, anche il diritto ospedaliero, o meglio la gestione degli enti ospedalieri ecclesiastici e secolari ${ }^{27}$. Ecco che nell'epoca della modernità giuridica il principe, all'interno di un'opera di razionalizzazione ed eliminazione costante e sistematica delle plurime realtà ordinamentali di matrice medievale - adombranti il suo disegno totalizzante e assolutistico - crea il modello organizzativo 'ospedale cittadino'. Fra i più importanti merita di essere ricordato l'Ospedale Sforzesco di Milano ${ }^{28}$, statutariamente nato nella metà del quattordicesimo secolo e modello di ospedali cittadini in altre città (Firenze, Mantova, Pavia, Lodi) ${ }^{29}$. L'operazione del principe moderno, come sottolineato da Nasalli Rocca, volta alla creazione di ospedali unitari - i cosiddetti Ospedali grandi - eliminando piccole realtà più o meno rurali, se da una parte è mossa dalla 'efficienza di governo nell'impresa sanitaria', per riprendere le parole che danno il titolo a questo nostro incontro, dall'altra non cela il vero e più profondo obiettivo consistente nella eliminazione della Chiesa dal controllo e dalla gestione dell'attività assistenziale sanitaria. Eliminazione non completamente realizzata a fronte di un modello di partecipazione mista, o compartecipazione, nella gestione degli enti ospedalieri che prevedeva l'esistenza di organi di gestione formati sia da esponenti della gerarchia ecclesiastica sia da quelli di organismi laici ${ }^{30}$.

\footnotetext{
${ }^{27}$ E. Nasalli Rocca, Il diritto ospedaliero nei suoi lineamenti storici, pp. 11-12.

${ }^{28}$ Ivi, pp. 81-88.

${ }^{29} \mathrm{Ivi}$, pp. $12-13$.

${ }^{30} I v i$, pp. 13-16; pp. 88-94.
} 
All'interno dell'operazione di rafforzamento dello Stato caratterizzante l'epoca moderna, dunque, si assiste in modo direttamente proporzionale alla volontà sempre più forte del principe di voler governare l'ente ecclesiastico ospedaliero nei confronti del quale eserciterà forti pressioni affinché nel regime di compartecipazione sia comunque preponderante la presenza di amministratori laici a scapito di quelli ecclesiastici. Ma la Storia, con i suoi eventi, serba sempre grandi sorprese e ciò che sembrava destinato ad essere un triste epilogo per il ruolo della Chiesa nello svolgimento dell'attività assistenziale ospedaliera si trasforma in una imponente reazione della Chiesa alla Riforma protestante ${ }^{31}$ che determina, dal nostro angolo di osservazione, nuovi spunti di riflessione: faccio riferimento al Concilio di Trento ${ }^{32}$, uno dei grandi

\footnotetext{
${ }^{31}$ Riguardo alla Riforma protestante si veda L. von Ranke, Deutsche Geschichte im Zeitalter der Reformation, 6 voll., Duncker und Humblot, Berlin 1839-1847; F. von Bezold, Storia della Riforma in Germania, Stab. Tip. Della Società Editrice Libraria, Milano 1900; E. GotHeIn, Schriften zur Kulturgeschichte der Renaissance, Reformation und Gegenreformation, II, Reformation und Gegenreformation, Duncker und Humblot, München 1924; P. Imbart DE La Tour, Origines de la Réforme, II, L'Église catholique: la crise et la renaissance, Librarie D'Argences, Melun 1946; E. G. LÉONARD, Histoire générale du Protestantisme, 3 voll., Presses universitaires de France, Paris 1961-1964; P. Tillich, L'era protestante, Claudiana, Torino 1972; K. Aland, Geschichte der Christenheit, II voll., Gütersloher Verl.-Haus Mohn, Gütersloh 1980-1982; S. H. HENDrIx, Tradition and authority in the Reformation, Ashgate variorum, Aldershot 1996; F. MussGnUG, Lutero e la riforma protestante, Giunti, Firenze 2003; D. K. McKIm, The Cambridge Companion to Martin Luther, Cambridge University Press, Cambridge 2003; R. H. BAInTon, Lutero e la riforma protestante, Mondadori, Milano 2011; M. NACCI, Chiesa e Stato dalla potestà contesa alla sana cooperatio. Un profilo storico-giuridico, Lateran University Press, Città del Vaticano 2015, pp. 14-15.

${ }^{32}$ Sul Concilio di Trento e il diritto tridentino si veda, H. Jedin, Geschichte des Konzils von Trient, 5 voll., Herder, Freiburg 1957-1977; G. Alberigo (ed.), Conciliorum Oecumenicorum Decreta, Istituto per le Scienze Religiose, Bologna 1973, pp. 657-799; A. Fliche - V. Martin (edd.), Storia della Chiesa, XVII, La Chiesa all'epoca del Concilio di Trento (1545-1563), di L. Cristiani, SAIE, Torino 1981; G. LE Bras - J. Gaudemet (ed.), Histoire du droit et des institutions de l'Église en Occident, XIV, L'époque de la Réforme et du Concile de Trente, di J. Bernhard-Ch. Lefebvre-F.
} 
concili ecumenici dell'epoca moderna che ci offre un'interessante lettura per lo sviluppo dell'ente ecclesiastico ospedaliero. Ed infatti, se da una parte i padri conciliari richiamano la supremazia della Chiesa sugli enti ecclesiastici ospedalieri attraverso precise regole (potere di vigilanza degli Ordinari sugli enti ecclesiastici ospedalieri, diritto di visita, diritto di controlli amministrativi e contabili), dall'altra, in pratica, non fanno altro che riprendere la normativa che fino allora si era stabilita in tema di gestione dell'ente ecclesiastico ospedaliero, quella della compartecipazione ${ }^{33}$. E questo per due ordini di ragioni: di fatto, sempre di più vi erano amministratori laici all'interno degli enti ecclesiastici ospedalieri e, in secondo luogo, perché vigeva il c.d. principio della protezione regia secondo il quale il re era protettore, a livello giuridico, di tali enti ${ }^{34}$.

La Chiesa, però, non si esprime solo attraverso la normativa del Concilio di Trento e grazie ad un sensus fidelium indirizzato verso la partecipazione reale all'assistenza dà vita ad importanti Ordini religiosi che si occupano principalmente di attività assistenziale ospedaliera. Due di essi meritano senz'altro di essere menzionati: l'Ordine Ospedaliero dei Fatebenefratelli, fondato a Granada da San

Rapp, Sirey, Paris 1989; G. Martina, La Chiesa nell'età dell'assolutismo, del liberalismo, del totalitarismo, I, L'età della riforma, Morcelliana, Brescia 1990, pp. 173200; M. Venard, Il Concilio Lateranense V e il Tridentino, in: G. Alberigo (ed.), Storia dei Concili Ecumenici, Queriniana, Brescia 1990, pp. 321-368; J. GAUdEMET, Storia del diritto canonico. Ecclesia et Civitas, San Paolo, Cinisello Balsamo 1998, pp. 711-716; A. Prosperi, Il Concilio di Trento: una introduzione storica, G. Einaudi, Torino 2001; P. Prodi, Il paradigma tridentino: un'epoca della storia della Chiesa, Morcelliana, Brescia 2010; J. W. O’Malley, Trento: il racconto del Concilio, traduzione di Stefano Galli, V\&P, Milano 2013; M. NACCI, L'evoluzione storica del diritto canonico e delle sue fonti giuridiche, in: M. J. Arroba Conde (ed.), Manuale di diritto canonico, Lateran University Press, Città del Vaticano 2014, p. 38; M. J. ArrobA Conde - M. Riondino, Introduzione al diritto canonico, Le Monnier UniversitàMondadori education, Firenze 2015, pp. 9-10; M. NACCI, Chiesa e Stato dalla potestà contesa alla sana cooperatio. Un profilo storico-giuridico, Lateran University Press, Città del Vaticano 2015, pp. 16-18.

${ }^{33}$ E. NAsalli Rocca, Il diritto ospedaliero nei suoi lineamenti storici, pp. 131-134. ${ }^{34}$ Ivi, p. 13. 
Giovanni di Dio (elevato ad ordine religioso nel 1586 da Sisto V), che attraverso il carisma della ospitalità si occupa della cura dei malati e dei bisognosi ${ }^{35}$; e l'Ordine dei Ministri degli Infermi, fondato da Camillo de Lellis ed elevato ad ordine religioso nel 1591 da Gregorio $\mathrm{XIV}^{36}$. Due esempi che sicuramente evidenziano la volontà della Chiesa di non abdicare alla funzione assistenziale ospedaliera della quale essa è da sempre portatrice e fedele interprete.

Sia con il Concilio di Trento sia, soprattutto, nell'epoca immediatamente successiva, la Chiesa prova a riaffermare una supremazia sugli enti ospedalieri attraverso le norme dei Sinodi diocesani e dei Concili provinciali ma, di fatto, in armonia con le disposizioni tridentine che in ogni caso prevedevano, come ho avuto modo di osservare, sia un'imponente presenza dei laici fra gli amministratori degli ospedali sia la protezione regia degli stessi enti ospedalieri tanto da delineare il già citato regime gestionale della compartecipazione ${ }^{37}$, peraltro confermato dalla dottrina canonistica e civilistica sei-settecentesca ${ }^{38}$.

${ }^{35}$ Cfr. sito internet dell'Ordine Ospedaliero di San Giovanni di Dio: http://www. ohsjd.org (consultato il 20 novembre 2015).

${ }^{36} \mathrm{Cfr}$. sito internet dell'Ordine dei Ministri degli Infermi: http://www.camilliani. org (consultato il 20 novembre 2015).

${ }^{37}$ E. Nasalli Rocca, Il diritto ospedaliero nei suoi lineamenti storici, pp. 135-138.

${ }^{38} \mathrm{Si}$ pensi, ad esempio a canonisti quali il Card. Domenico Toschi che nell'opera Practicarum Conclusionum iuris in omni foro frequentiorum (1621) affermava che l'ospedale per essere tale necessitava della licenza del Vescovo ma potevano esistere anche ospedali laici; oppure al Card. G. B. De Luca che nel suo Theatrum Veritatis et Justitiae (dal Repertorium del 1726) insisteva sul fatto che gli ospedali ecclesiastici dovevano essere regolati iure universitatum ma che i beni che il principe donava all'ospedale erano pubblici e che il popolo era interessato al buon andamento dell'ospedale del quale doveva interessarsi, dal punto di vista amministrativo e gestionale, il principe insieme al vescovo; ancora, lo spagnolo Francesco Mostazo nel De causis piis (1721) distingueva gli ospedali ecclesiastici, luoghi pii fondati dal vescovo e con gli stessi privilegi delle chiese, da quelli profani fondati invece da privati che non comportavano un intervento vescovile (E. NASALli Rocca, Il diritto ospedaliero nei suoi lineamenti storici, pp. 162-180). Fra i civilisti, invece, merita attenzione M. A. Sabelli e la sua Summa diversorum tractatuum, una vera e propria opera di ius commune in tema di diritto ospedaliero, dove si intrecciano 
Il regime della compartecipazione, però, viene svuotato di contenuto nell'epoca rivoluzionaria francese attraverso concetti quali l'obbligo-diritto di assistenza a cura dello Stato ${ }^{39}$ e normative tendenti ad una completa laicizzazione dell'amministrazione dell'ente ospedaliero, esautorando così il vescovo del preminente ruolo direttivo e di controllo di cui era stato titolare nelle epoche precedenti ${ }^{40}$. In ogni caso la Chiesa, attraverso la prima codificazione del diritto canonico ${ }^{41}$, ha comunque affermato 'a chiare lettere' il «suo diritto di legiferare e di vigilare l'amministrazione degli enti di beneficienza con carattere di alta superiorità», così come emerge chiaramente nei canoni 14891494 dove vengono delineate le norme di diritto canonico universale in materia di enti benefici ospedalieri ${ }^{42}$.

sapientemente fonti civilistiche e fonti canonistiche; e il pistoiese G. B. Pacichelli con il suo De iure hospitalitatis universo, manuale completo ed esaustivo di diritto ospedaliero dove si tratta, ad esempio, del c.d. ius metatorum, dei privilegi ospedalieri, degli amministratori, degli ospedali speciali (E. NASALli RoccA, Il diritto ospedaliero nei suoi lineamenti storici, pp. 181-203).

${ }^{39}$ E. NAsAlli Rocca, Il diritto ospedaliero nei suoi lineamenti storici, p. 217.

${ }^{40}$ Ivi, p. 220.

${ }^{41}$ Sulla codificazione piano-benedettina si veda, ex multis, G. FeliCiAni, Il Concilio Vaticano I e la codificazione del diritto canonico, Giuffrè, Milano 1982; G. Feliciani, Il cardinal Gasparri e la codificazione del diritto canonico, in: M. Tedeschi (ed.), Studi in onore di Gaetano Catalano, II, Rubbettino, Soveria Mannelli 1998, pp. 563-579; C. Fantappiè, Pio X e il “Codex iuris canonici”, in: A. Cattaneo (ed.), L'eredità giuridica di San Pio X, Marcianum Press, Venezia 2006, pp. 155-171; C. FAntAPPIÈ, Chiesa romana e modernità giuridica, II, Il Codex iuris canonici (1917), Giuffrè, Milano 2008; P. Grossi, Valore e limiti della codificazione del diritto (con qualche annotazione sulla scelta codicistica del legislatore canonico), in: ID., Scritti canonistici, (C. Fantappiè, ed.), Giuffrè, Milano 2013, pp. 247-263; P. GHERRI, Codificazione canonica tra tecnica e sistema, in: Eastern Canon Law, 2 (2013), pp. 19-130; C. Minelli, Pio X e l'avvio del processo di codificazione, in: Stato, Chiese e pluralismo confessionale. Rivista telematica (www.statoechiese.it), 33 (2013), pp. 1-38; M. Nacci, San Pio X e il diritto canonico: la "cultura giuridica" della codificazione del diritto della Chiesa, Ephemerides Iuris Canonici 54 (2014), pp. 87-101.

${ }^{42}$ Codex Iuris Canonici, 1917, Lib. III, De rebus, Pars V, De beneficiis aliisque institutis ecclesiasticis non collegialibus, Tit. XXVI, De aliis institutis ecclesiasticis non collegialibus, can. 1489: $\$ 1$. Hospitalia, orphanotrophia, aliaque similia instituta, ad opera religionis vel caritatis sive spiritualis sive temporalis destinata, possunt ab 


\section{Nota conclusiva}

Da quanto abbiamo potuto osservare in questo breve excursus storico-giuridico, credo si possa rilevare che circa l'esercizio dell'assistenza e, nello specifico, sul versante dello sviluppo dell'ente ecclesiastico ospedaliero, la Chiesa e lo Stato si sono incontrati o scontrati, secondo le diverse epoche storiche, in base al loro ruolo più o meno 'protagonistico' svolto nella società. È al tempo stesso indubbio, come osserva Dalla Torre, che il maggior ruolo della Chiesa nello svolgimento dell'attività assistenziale ospedaliera all'interno della compagine umana si sia determinato sulla base di una "coscienza comune" secondo cui «le opere di misericordia, fra cui quelle di misericordia corporale, in quanto esercizio della carità

Ordinario loci erigi et per eius decretum persona iuridica in Ecclesia constitui. \$2. Loci Ordinarius haec instituta ne approbet, nisi finis fundationis utilis reapse sit, et talis constituta fuerit dos, quae, omnibus perpensis, sufficiat vel sufficiens fore prudenter praevideatur ad illum assequendum. $\$ 3$. Horum institutorum administrare bona sui cuiusque rectroris est, secundum normas tabulae fundationis; hic vero iisdem obligationibus tenetur, iisdemque iuribus fruitur, quibus administratores aliorum bonorum ecclesiasticorum; can. 1490: $\$ 1$. In tabulis fundationis pius fundator accurate describat totam instituti constitutionem, finem, dotationem, administrationem et regimen, usum redituum et successionem in bona, casu exstinctionis ipsius instituti. $\$ 2$. Huiusmodi tabulae duplici exemplari conficiendae sunt, quorum alterum in archivo instituti, alterum in archivo Curiae reponatur; can 1491: $\$ 1$. Loci Ordinarius omnia huiusmodi instituta, etiam in personam moralem erecta et quovis modo exempta, visitare potest et debet. $\$ 2$. Imo licet in personam moralem non sint erecta et domui religiosae concredita, si quidem agatur de domo religiosa iuris dioecesani, iurisdictioni Ordinarii loci penitus subduntur; si de domo religiosa iuris pontificii, episcopali vigilantiae subsunt quod spectat ad religionis magisteria, honestatem morum, exercitationes pietatis, sacrorum administrationem; can. 1492: \$1. Etiamsi fundatione, praescriptione aut privilegio apostolico pium institutum a iurisdictione et visitatione Ordinarii loci exemptum fuerit, ius tamen Ordinario est redditionem rationum exigendi, reprobata contraria consuetudine. $\$ 2$. Si fundator velit administratores non teneri rationem Ordinario loci reddere, fundatio ne acceptetur; can. 1493: Ordinarius loci advigilet ut piae fidelium voluntates, in horum institutorum fundatione expressae, plene serventur; can. 1494: Sine venia Sedis Apostolicae haec instituta supprimi, uniri, vel in usus a fundatione alienos converti nequeunt, nisi in tabulis fundationis aliud caveatur. 
cristiana e strumento di spirituale perfezione personale, rientrassero certamente nell'ambito delle competenze proprie della Chiesa ${ }^{43}$. Una coscienza comune che sono convinto debba essere recuperata, e in molti casi purtroppo completamente riacquisita, da questa società del terzo millennio che troppo spesso è proiettata a ingigantire la sua dimensione individualistica dimenticando, invece, quella collettiva e comunitaria.

\section{The model "ecclesiastical institution": the historical perspective}

This work is a conference in the Faculty of Law of the University of Bari (Taranto Department, Italy) that consider the historical-juridical evolution of the "ecclesiastical institution" model with respect to the welfare activity carried out by the Church and to the ecclesiastical hospital institution.

Parole chiave: diritto canonico; storia del diritto; "ente ecclesiastico"; attività assistenziale; ente ecclesiastico ospedaliero

KEY wORDS: Canon law; legal history; "ecclesiastical institution"; history of Law; Church's welfare activity; ecclesiastical hospital institution

\section{Nota o Autorze:}

Prof. Matteo Nacci - is full professor of History of Law and director of Department of History of Law in the Pontifical Lateran University.

\footnotetext{
${ }^{43}$ G. Dalla Torre, L'attività assistenziale della Chiesa nell'ordinamento italiano. Aspetti dogmatici e spunti ricostruttivi, p. 37.
} 